\title{
ExprAlign - the identification of ESTs in non-model species by alignment of cDNA microarray expression profiles Weizhong Li1 ${ }^{1,4}$, Andrew Y Gracey ${ }^{1,2}$, Luciane Vieira Mello ${ }^{1}$, Andrew Brass ${ }^{3}$ and Andrew R Cossins*1
}

\author{
Address: ${ }^{1}$ Centre for Genome Research, School of Biological Sciences, University of Liverpool, Crown Street, Liverpool, L69 7ZB, UK, ${ }^{2}$ Marine \\ Environmental Biology, University of Southern California, Los Angeles, CA 90089, USA, ${ }^{3}$ Computing Science Department, University of \\ Manchester, Kilburn Building, Oxford Road, Manchester, M13 9PL, UK and ${ }^{4}$ EMBL - European Bioinformatics Institute, Wellcome Trust Genome \\ Campus, Hinxton, Cambridge, CB10 1SD, UK \\ Email: Weizhong Li -w.li@ebi.ac.uk; Andrew Y Gracey - gracey@usc.edu; Luciane Vieira Mello - lumello@liverpool.ac.uk; \\ Andrew Brass - a.brass@manchester.ac.uk; Andrew R Cossins* - cossins@liverpool.ac.uk \\ * Corresponding author
}

Published: 26 November 2009

BMC Genomics 2009, 10:560 doi:10.1186/147|-2/64-10-560
Received: 21 January 2009

Accepted: 26 November 2009

This article is available from: http://www.biomedcentral.com/I47I-2/64/I0/560

(C) 2009 Li et al; licensee BioMed Central Ltd.

This is an Open Access article distributed under the terms of the Creative Commons Attribution License (http://creativecommons.org/licenses/by/2.0), which permits unrestricted use, distribution, and reproduction in any medium, provided the original work is properly cited.

\begin{abstract}
Background: Sequence identification of ESTs from non-model species offers distinct challenges particularly when these species have duplicated genomes and when they are phylogenetically distant from sequenced model organisms. For the common carp, an environmental model of aquacultural interest, large numbers of ESTs remained unidentified using BLAST sequence alignment. We have used the expression profiles from large-scale microarray experiments to suggest gene identities.
\end{abstract}

Results: Expression profiles from $\sim 700$ cDNA microarrays describing responses of 7 major tissues to multiple environmental stressors were used to define a co-expression landscape. This was based on the Pearsons correlation coefficient relating each gene with all other genes, from which a network description provided clusters of highly correlated genes as 'mountains'. We show that these contain genes with known identities and genes with unknown identities, and that the correlation constitutes evidence of identity in the latter. This procedure has suggested identities to 522 of $270 \mathrm{I}$ unknown carp ESTs sequences. We also discriminate several common carp genes and gene isoforms that were not discriminated by BLAST sequence alignment alone. Precision in identification was substantially improved by use of data from multiple tissues and treatments.

Conclusion: The detailed analysis of co-expression landscapes is a sensitive technique for suggesting an identity for the large number of BLAST unidentified cDNAs generated in EST projects. It is capable of detecting even subtle changes in expression profiles, and thereby of distinguishing genes with a common BLAST identity into different identities. It benefits from the use of multiple treatments or contrasts, and from the large-scale microarray data.

\section{Background}

Transcript screening investigations have traditionally been led by sequence analysis of cDNA clone collections to define the identity of hybridisation probes included on microarrays for expression profiling [1]. Despite this, all eukaryotic EST collections contain large proportions of 
transcripts $(\sim 50 \%)$ that remain unidentified by unattended BLAST protocols. Some of these may represent new, undiscovered protein-coding or non-protein-coding transcripts [2-4]. Others may arise from untranslated regions of coding sequence RNA, which being non-conserved fail to align to reference databases. Finally, some may be concatenated constructs generated artefactually during the production of cDNA libraries.

We have experienced these kinds of difficulty in our analysis of ESTs from the common carp, Cyprinus carpio L., a well-used model species for research into environmental responses [5], and which is the subject of a substantial aquaculture interest for both food and ornamental uses. The common carp genome is widely thought to have become duplicated within the previous 12-15 Mya, and many duplicate paralogs are retained [6-8] to complicate the analysis. We originally generated a medium-scale collection of $\sim 13.5 \mathrm{~K}$ directional, cDNA clones from multiple tissues [9], though this has more recently been increased [10]. 9,202 directional EST were assembled into 6,033 transcriptional units. Of these only 3,252 were BLASTidentified leaving 2,701 as unclassified, many of which displayed interesting expression properties in response to a range of chronic stress treatments [9].

Additional information regarding the identity of ESTs may come from the comparison of expression profiles of one microarray probe with another since different probes arising from the same gene should have very highly correlated profiles whilst probes with an identical BLAST identity but arising from different members of a gene family might present divergent expression profiles. Either way, co-expression indices can be used as evidence in seeking an identity for a BLAST-unidentified cDNA clone, and can separate putative isoforms. To explore the limits of expression profiling, and the extent to which dissimilar but coregulated genes may confound the process, we have accumulated data from a very large number of microarray hybridisations, including RNA from all of the major organs of common carp exposed to a range of environmental stresses, including chronic cooling [9], chronic hypoxia [11] and starvation/refeeding protocols. This large dataset represents a substantial data resource that can be used to suggest gene identity through correlation analysis. Here we describe the Expression Alignment (ExprAlign) technique for assigning a putative gene identity, which, following the pioneering work of Kim et al. $[12,13]$, is based on the clustering of gene expression profiles [14-17]. This resolves a number of issues relating to the identification of probes that were unidentified by conventional unattended BLASTx procedures, including those from untranslated regions of transcripts.

\section{Methods}

\section{ESTs resource and common carp microarray data}

We used the EST resources from carpBASE 2.1, which was constructed by the EST analysis package EST-ferret 2.1 http://legr.liv.ac.uk. This comprised the 13,349 directional cDNA clones already described [10], of which 9202 were 5 ' end sequenced, BLASTx identified and annotated with gene ontology, KEGG and CDD terms.

The cDNA microarray used in this work has been described in [9] and [11], and comprised 13440 PCRamplified cDNA probes, including blanks and standards. The raw expression data has been deposited in ArrayExpress E-MAXD-1 and E-MAXD-10, respectively. The gene expression data used in this analysis comprised 707 common carp RNA samples, hybridised to 1414 cDNA microarrays, all using a reference-based, dye-swap design against a common reference using dye-swap, and with 4fold or greater biological replication. These experiments were conducted with ethical approval and corresponding personal and project licences of the Home Office, U.K 189 RNA samples were generated from the study of chronic cold stress [9], including samples for time-course after transfer from a preconditioning temperature of $30^{\circ} \mathrm{C}$ to $23^{\circ} \mathrm{C}, 17^{\circ} \mathrm{C}$, and $10^{\circ} \mathrm{C}$. Tissues examined were brain, gill, heart, intestine, kidney, liver and skeletal muscle. 414 RNA samples were used in a time-course study of hypoxia stress [11] in 4 tissues (brain, heart, intestine, liver and skeletal muscle), conducted at two temperatures $\left(17^{\circ} \mathrm{C}\right.$ and $30^{\circ} \mathrm{C}$ ). 104 samples were from a study of starvation over a 6 -week time course during which the animals were starved and then re-fed (I. Hardevig and A.R. Cossins, unpublished). The 'starvation' data included samples for liver and skeletal muscle only. Normalisation and statistical processing of the resulting data has been previously described [9,11] using established techniques [18].

\section{Computing and filtering Pearson's correlation coefficients}

The Pearson product-moment correlation coefficient $(r)$ was calculated for every pair of array probes. To speed this up a programme called "CORR" http://legr.liv.ac.uk was written in C, resulting in completion in approximately 40 minutes using a single Linux machine. The Receiver Operating Characteristic (ROC) curves were implemented as described in Additional File 1, Section A, to optimize the threshold of the correlations [19]. The resulting criterion was used to filter the $r$-values, and the selected relationships were stored in a spreadsheet consisting of a few thousand gene-pairs (the rows), with coefficient scores.

The optimized threshold for filtering Pearson correlation coefficients was determined by plotting the sensitivity (True Positive, $\mathrm{P}^{+}$) of the comparison against the selectivity (False Positive, $\mathrm{P}^{-}$). The sensitivity $\mathrm{P}^{+}$indicates the probability of the observed true positives at a threshold 
and the selectivity $\mathrm{P}$ - shows the probability for the observed true negatives at a threshold. On the other hand, the probability of the missed true positives was given by $\left(1-\mathrm{P}^{+}\right)$and the probability of the missed true negatives by (1 - P-). The total probability of missing the true positives and true negatives was given by $\mathrm{E}=\left(1-\mathrm{P}^{+}\right)+\left(1-\mathrm{P}^{-}\right)$, this providing the optimal threshold. The calculations for $\mathrm{P}$ and $\mathrm{E}$ are detailed in the Additional File 1, Section A.

\section{Visualising expression alignments}

The VxInsight package [20-22] was implemented for clustering gene expressions using the Pearson's correlation coefficients and to visualise the alignments. The VxInsight package consists of three parts: VxOrd, VxInsight and VxImport. VxOrd implements the force-directed ordination algorithm to assign the $\mathrm{X}, \mathrm{Y}$ coordinates for each gene on a 2-dimensional surface based on the clone pair similarities of correlation coefficients. Then the coordinate maps were converted to the 3-dimensional mountain terrains in VxInsight. Finally, VxImport loaded the gene annotation from carpBASE 2.1 into VxInsight for biological interpretations.

Figure 1 illustrates the stages used in ExprAlign to generate a landscape depiction of the expression profile, starting from the microarray intensity data and ending with the production of the colour-rendered 3D landscape.

\section{Post ExprAlign analysis}

Groups of cDNA clones included within landscape features were assessed using BLASTx, searches as described previously [10] followed by mapping of FASTA sequences onto the zebrafish genome v8 as described by Christoffels et al. [23], or onto common carp fosmid clones BX571686 and BX571725 [24]. Details of Pearsons correlation coefficient of closely related clones, and of mountain allocations, have been included as part of the clone entry in carpBASE2.1.

\section{Results}

\section{Correlation analysis}

Using normalised data generated from 707 RNA preparations from the cold and hypoxia datasets, each containing $\sim 13,440$ cDNA probes from multiple tissues we calculated the Pearson correlation coefficient [25] between every pair of probes represented on the microarray. This required 180 million calculations, covering different combination of stress and time, as well as all different tissues. We then used a Receiver Operating Characteristic (ROC) procedure [19] to optimize the threshold that minimises the representation of false positives at 0.858 . The coefficients below this value were discarded leaving 30868 correlation coefficients representing 3039 gene probes for construction of the landscape.

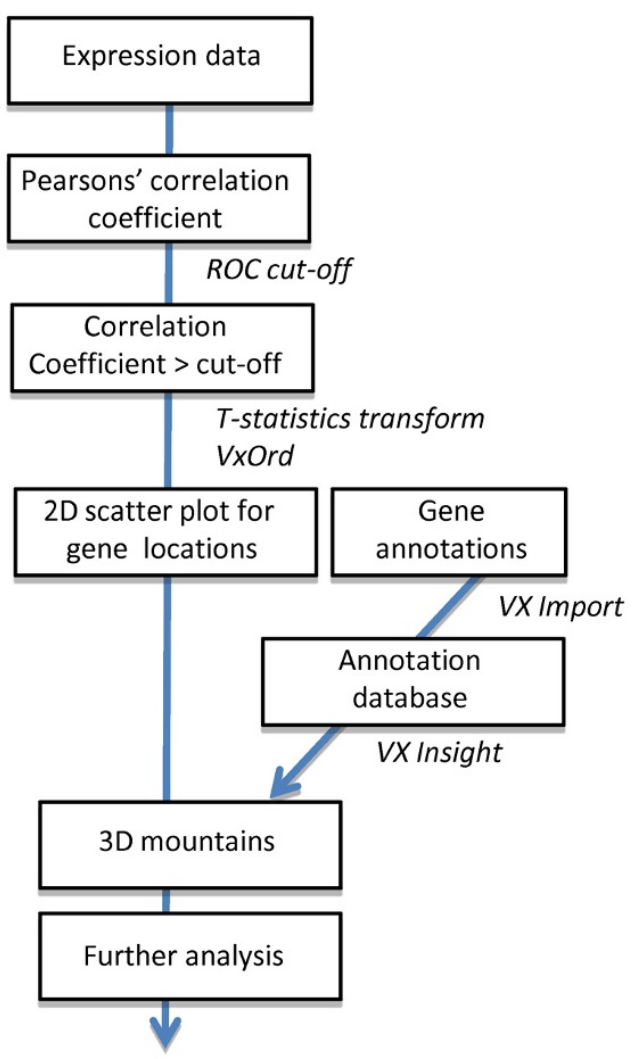

Figure I

Flow diagram depicting the order of events (in boxes) and informatic tools and packages used (italics) in the ExprAlign pipeline.

\section{Network analysis using 3D landscape for visualisation}

We then created a network representation of the $r$-values that exceeded the ROC threshold, which was ordinated and visualized using VxInsight [20]. This clustered groups of gene probes with high $r$-values between them using a force-repulsion model and represented them as features in a 3-dimensional landscape metaphor, as an aid to easy interpretation (see Figures $2 \mathrm{a}$ and $2 \mathrm{~b}$ ). The height of each landscape feature was an indication of the number of gene probes contained within it, and large-scale features ('mountains', 'hills') can include a number of smaller features ('hillocks'). The 3039 gene probes previously identified comprised 1192 possessing a BLASTx identity whilst the remaining 1847 were unclassifiable. Big mountains were located around the centre of the landscape while smaller mountains were positioned away from the centre. The distance between each feature, both large and small, was representative of the $r$-values that connect the features. Thus a small distance between landscape features 

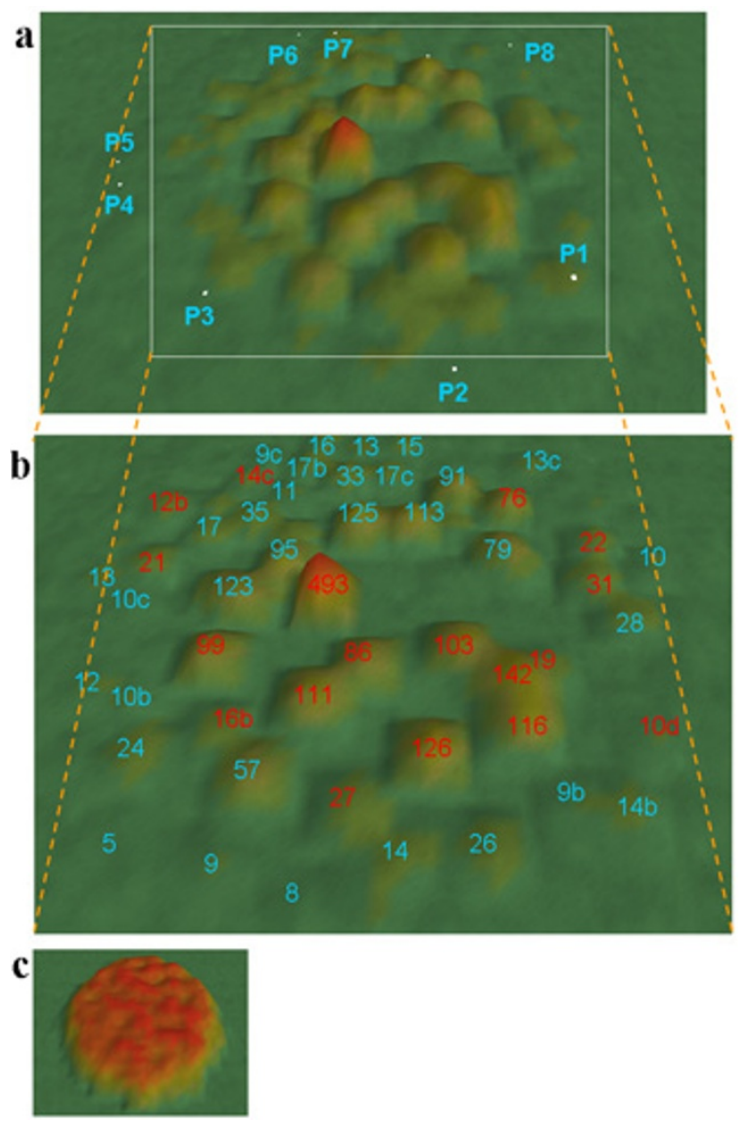

Figure 2

A landscape representation of the co-expression profile for the GE analysis of common carp genes. (a) An overview landscape. The white spots, PI to P8, represent the locations of different parvalbumin isoforms. (b) shows a magnified part of (a) showing labels for each feature displaying the number of clones within that feature. 'Identified' mountains were indicated in blue text and 'unknown' mountains in red. (c) Terrain map derived from permuted and thus random-shuffled array data.

indicates that each cluster of gene probes was more similar to each other than if they were more distantly linked.

To assess the significance of the topographical patterns revealed by this process, we permuted the expression table by shuffling the values for all probes across all arrays. The resulting $r$-values were uniformly low with only 3 pairs of probes with $r>0.858$, and 33870 with $r>0.25$. We used the latter data to generate a landscape shown in Figure 2c, which showed no structuring. This indicates that the landscape features evident in Figure 2a do not result from random effects in the underlying data, and represent biologically significant outcomes.

\section{Dependency of landscape features upon scale and diversity of datasets}

To determine whether the structure of the gene co-expression landscape and the clustering of genes was influenced by the particular dataset used in their construction, we recalculated the landscape using only the data from the 386 arrays generated for the cooling experiment [9]. In this case 4236 microarray probes exceeded the ROC threshold and were ordinated onto the landscape map, comprising 1776 identified and 2460 unclassifiable gene probes. 46 mountains (labeled 'CE', 'cold expression') were generated of which 22 were identified.

We then compared the landscape features for both the GE and CE datasets (provided in reduced form in Figure 3, and shown in full in Additional File 1, Figure S1) using a matrix in which cells contained the number of gene probes that were included in corresponding landscape features. We found 21 highly similar mountain-pairs between GE and CE mountains, indicated by greyed cells. However, we noted some differences between the two landscapes. Thus, the clones in mountain CE209 were separately located in GE142 and GE103; clones in CE178 were separately located in GE111 and GE16b; and the clones in CE149 were separately located in GE142 and GE116. Feature CE26 (see Additional File 1, Figure S1) was linked to three GE features, namely GE35 (apolipoprotein A-I), GE17 (transferrin) and GE13 (transferrin). Moreover, CE119 (creatine kinases) was linked to GE57 and GE24 (both labelled as creatine kinases); and CE121 (parvalbumins) were broken down to GE16 and GE14b (both parvalbumins). The splitting of CE features into two GE features might indicate the separation of isoforms.

We conclude that the discrimination between closely linked gene clusters was significantly affected either by the scale or by the diversity of the data used in their construction. We then tested which of these was the important factor by randomly discarding $50 \%$ of the arrays included in the GE dataset. The resulting landscape contained 2444 clones in 27 mountains (labelled 'RE', randomised expression) of which 12 were identified. We then compared the contents of landscape features of the RE dataset with the original GE dataset (summarised in Figure 4 and displayed in full in Additional File 1, Figure S2). This shows that 17 of the RE mountains were related to single features on the GE landscape, and that the 'identity' of these features was conserved. However, 11 of the RE mountains were linked to multiple GE mountains; for example, RE237 and RE174 were each linked to 3 different features in the GE landscape. Thus, whilst the main features of the landscape were robust to reductions in the amount of array data some were sensitive to increasing data complexity, as produced by undertaking more diverse experimental treatments. 


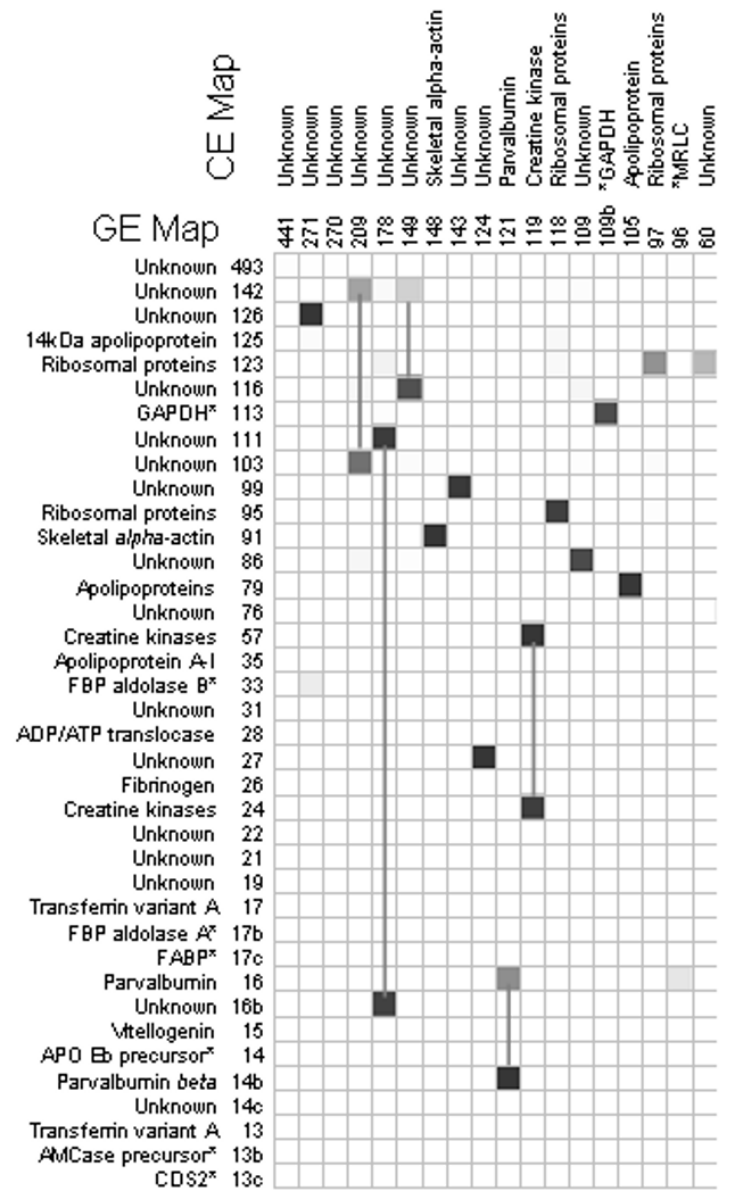

Figure 3

Matrix to compare the distribution of selected gene identities in GE and CE landscape co-expression features. Additional File I, Figure SI comprises all features. The labels for each column or row indicates the number of genes within that landscape feature. The GE features are listed in Table I. The numbers contained within individual cells indicate the number of microarray probes common to the linked GE and CE features. The denser colour represents a greater level of the agreement.

\section{Relating unidentified or unclassifiable clones to identified genes}

To test the significance of the representation of identified genes within each of the landscape features we computed $p$-values for the GE dataset for each gene identity using Fisher's exact test. Table 1 lists the p-values, all of which lay between $10^{13}$ and $10^{101}$, indicating highly significant enrichment of the identified gene within the mountain.

Secondly, we examined individual clusters either by attended curation of the ESTs followed by BLASTx or by mapping clones onto the zebrafish genome. Thus, for mountain GE13c, only 4 clones recovered a BLASTx hit for

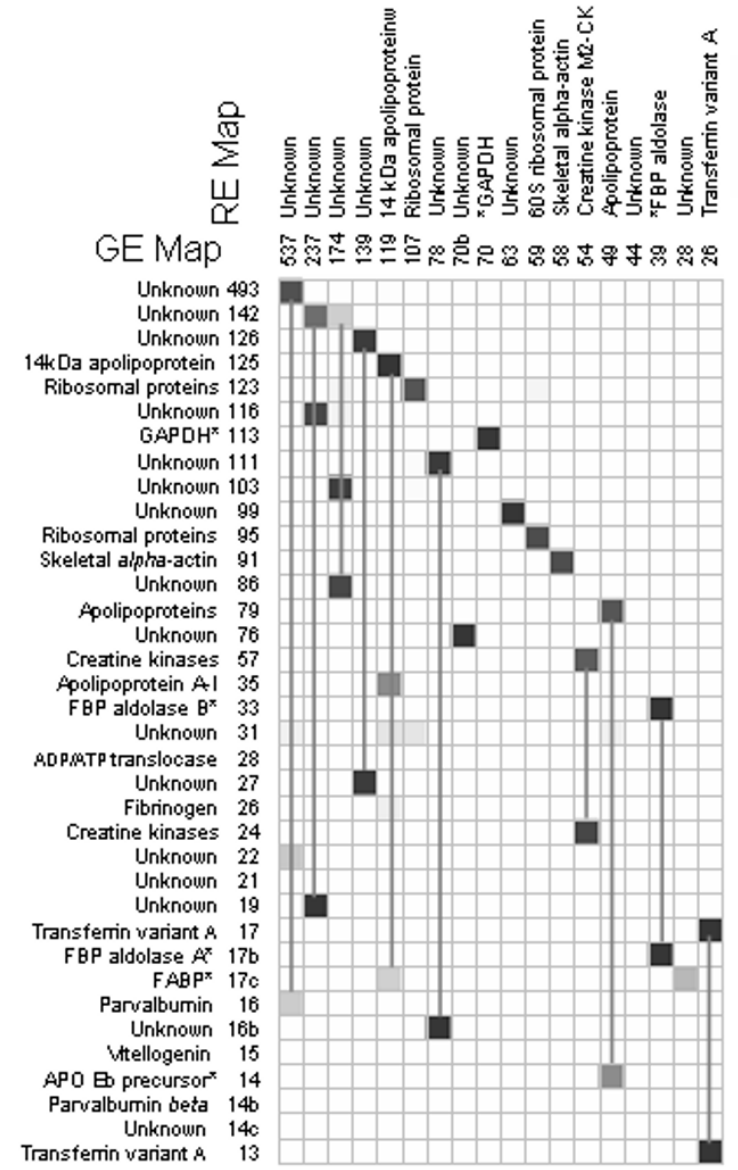

\section{Figure 4}

Matrix for agreement and difference of global mountains and random mountains. This figure includes selected landscape features whilst Additional File I, Figure S2 comprises all features. Other details are as described in the legend to Figure 3.

the common carp desaturase-2, 2 of them following a frame shift correction. A further 6 were confirmed as belonging to this gene using BLASTn against the 2 available common carp fosmid clones but in the untranslated region, two of them being very short sequence reads. The three remaining clones failed to provide a sequence, but were inferred as being the same gene. Mountain GE10d contained only unidentifiable sequences (thus, not included in the listing of identified mountains in Table 1), all of which belonged to the same carpBASE2.1 contig (1127-2) and which mapped to the same zebrafish location (Chromosome 23: 24767100). This lies within an unprocessed pseudogene (locus i.d. OTTDARG00000030773) so whilst the identity and status of this transcriptional product remains unclear, it is revealed as being expressed in particular common carp tissues and in response to particular environmental influ- 
Table I: Summary of landscape features identified for the complete (GE) dataset.

\begin{tabular}{|c|c|c|c|c|c|c|c|c|}
\hline \multirow{2}{*}{$\begin{array}{c}\text { GE } \\
\text { Mountain } \\
\text { i.d. }\end{array}$} & \multirow{2}{*}{$\begin{array}{l}\text { No. of } \\
\text { identified } \\
\text { clones }\end{array}$} & \multicolumn{6}{|c|}{ Best represented gene identities } & \multirow{2}{*}{$\begin{array}{c}\text { No. of } \\
\text { relatable } \\
\text { unknown } \\
\text { clones }\end{array}$} \\
\hline & & Protein description & $\begin{array}{l}\text { No. in } \\
\text { mountain }\end{array}$ & $\begin{array}{l}\% \text { of the } \\
\text { identified } \\
\text { clones in } \\
\text { mountain }\end{array}$ & $\begin{array}{l}\% \text { of clones } \\
\text { in mountain }\end{array}$ & $\begin{array}{c}\text { No. in } \\
\text { carpBASE } \\
2.1\end{array}$ & $\begin{array}{c}p \text { to the } \\
\text { carpBASE } \\
2.1\end{array}$ & \\
\hline 125 & 48 & I4 kDa apolipoprotein & 34 & 70.8 & 27.2 & 65 & $3.2 \mathrm{E}-60$ & 77 \\
\hline 123 & 53 & Ribosomal proteins & 39 & 73.6 & 31.7 & 339 & $5.9 \mathrm{E}-38$ & 70 \\
\hline 113 & 55 & $\begin{array}{l}\text { Glyceraldehyde-3-phosphate } \\
\text { dehydrogenase }\end{array}$ & 50 & 90.9 & 44.2 & 70 & $6.3 \mathrm{E}-101$ & 58 \\
\hline 95 & 23 & Ribosomal proteins & 19 & 82.6 & 20 & 339 & I.2E-20 & 72 \\
\hline 91 & 46 & Skeletal alpha-actin & 36 & 78.3 & 39.6 & 71 & $6.5 \mathrm{E}-65$ & 45 \\
\hline 79 & 39 & Apolipoproteins & 33 & 84.6 & 41.8 & 113 & $3.9 \mathrm{E}-53$ & 40 \\
\hline 57 & 24 & Creatine kinases & 20 & 83.3 & 35.1 & 74 & $5.9 \mathrm{E}-36$ & 33 \\
\hline 35 & 15 & Apolipoprotein A-I & 13 & 86.7 & 37.1 & 47 & $8.9 E-27$ & 20 \\
\hline 33 & 28 & $\begin{array}{l}\text { Fructose-bisphosphate } \\
\text { aldolase B }\end{array}$ & 26 & 92.9 & 78.8 & 30 & 4.IE-65 & 5 \\
\hline 28 & 26 & ADP/ATP translocases & 26 & 100 & 92.9 & 43 & I.7E-60 & 2 \\
\hline 26 & 16 & Fibrinogen & 14 & 87.5 & 53.8 & 35 & $4.0 \mathrm{E}-3 \mathrm{I}$ & 10 \\
\hline 24 & 6 & Creatine kinases & 4 & 66.7 & 16.7 & 74 & 3.E-07 & 18 \\
\hline 17 & 9 & Transferrin variant $A$ & 8 & 88.9 & 47.1 & 44 & 4.IE-I7 & 8 \\
\hline $17 b$ & 15 & $\begin{array}{l}\text { Fructose-bisphosphate } \\
\text { aldolase A }\end{array}$ & 13 & 86.7 & 76.5 & 30 & $7.6 \mathrm{E}-30$ & 2 \\
\hline I7c & 13 & Fatty acid-binding protein & 13 & 100 & 76.5 & 28 & $2.3 \mathrm{E}-32$ & 4 \\
\hline 16 & 9 & Parvalbumins & 8 & 88.9 & 50 & 114 & $1.4 \mathrm{E}-13$ & 7 \\
\hline 15 & 6 & Vitellogenin & 6 & 100 & 40 & 10 & $3.6 \mathrm{E}-18$ & 9 \\
\hline 14 & 5 & Apolipoprotein Eb precursor & 5 & 100 & 35.7 & 19 & $2.0 \mathrm{E}-13$ & 9 \\
\hline I4b & 14 & Parvalbumins & 14 & 100 & 100 & 114 & $4.5 \mathrm{E}-25$ & 0 \\
\hline 13 & 9 & Transferrin variant $\mathrm{A}$ & 7 & 77.8 & 53.8 & 44 & $2.8 \mathrm{E}-14$ & 4 \\
\hline $13 b$ & 7 & $\begin{array}{l}\text { Acidic mammalian chitinase } \\
\text { precursor }\end{array}$ & 7 & 100 & 53.8 & 11 & $6.8 \mathrm{E}-21$ & 6 \\
\hline $13 c$ & 10 & Carp Desaturase 2 (CDS2) & 10 & 100 & 76.9 & 15 & $2.2 \mathrm{E}-28$ & 3 \\
\hline 12 & 9 & $\begin{array}{l}\text { Troponin T, fast skeletal } \\
\text { muscle isoforms }\end{array}$ & 9 & 100 & 75 & 22 & 2.IE-23 & 3 \\
\hline II & 9 & $\begin{array}{l}\text { Apolipoprotein C-I } \\
\text { precursor }\end{array}$ & 9 & 100 & 81.8 & 24 & $5.6 \mathrm{E}-23$ & 2 \\
\hline 10 & 5 & Myoglobin & 5 & 100 & 50 & 6 & $1.0 \mathrm{E}-16$ & 5 \\
\hline $10 \mathrm{~b}$ & 8 & $\begin{array}{l}\text { Warm-temperature- } \\
\text { acclimation-related-65 kDa- } \\
\text { protein }\end{array}$ & 5 & 62.5 & 50 & 15 & $2.8 \mathrm{E}-12$ & 2 \\
\hline $10 c$ & 9 & Uncoupling protein I & 9 & 100 & 90 & 10 & $4.3 \mathrm{E}-28$ & 1 \\
\hline 9 & 8 & C-type lectin & 8 & 100 & 88.9 & 19 & 2.|E-2| & 1 \\
\hline $9 b$ & 9 & Invariant chain like protein 2 & 9 & 100 & 100 & 20 & 7.IE-24 & 0 \\
\hline $9 c$ & 6 & $\begin{array}{l}\text { Elongation factor I-alpha; } \\
\text { EF-I-alpha }\end{array}$ & 6 & 100 & 66.7 & 13 & $3.0 \mathrm{E}-17$ & 3 \\
\hline 8 & 6 & Alcohol dehydrogenase & 6 & 100 & 75 & 24 & $2.3 \mathrm{E}-15$ & 2 \\
\hline 5 & 4 & RING finger protein 28 & 4 & 100 & 80 & 6 & $3.0 \mathrm{E}-13$ & 1 \\
\hline Total & 549 & & & & & & & 522 \\
\hline
\end{tabular}

Column I represents the number of gene probes included within the landscape feature indicated in Figure 2, and this is also used to denote the identity. Column 2 indicates how many of these were BLAST identified as indicated in column 3 . The number of the best-represented identified gene in a mountain must be $>2$, and its percentage of clones in mountain must be over $20 \%$. $P$ represents the significance (Fishers Exact test) of the gene being over-represented in the mountain comparing to the whole clone set included within carpBASE 2.I. 
ences. Mountain GE10 contained 5 ESTs that were identified by BLASTx as myoglobin, and 5 ESTs that were frame shift corrected also to BLASTx as myoglobin. Finally, in mountain GE113, 50 of the 55 identified genes (i.e. 91\%) were BLASTed as glyceraldehyde 3-phosphate dehydrogenase. The $p$-value for this identity (6e-101) was very highly significant. However, 5 ESTs showed homology to different genes including microglobulin, adenylate kinse, 60S ribosomal protein, and a glycoprotein precursor. The remaining 58 clones lacking sequence data were thus inferred as having the same identity as the predominant gene.

For large mountains, in particular, we found multiple identified genes being represented, making it difficult to ascribe a single identity to unidentified clones. In some cases we have generated 3-5 sub-clusters using the Kmeans technique, each of which displayed a more singular gene identity with which to infer an identity for the unidentified or un-sequenced ESTs (see below).

Using these methods, we inferred a tentative identity for a total of 522 unidentified ESTs, which represented 17\% of the 3039 unidentified ESTs. These are listed in Additional File 1, Table S1. From this it is evident that many clones failed to generate sequence data during the automated sequence analysis, yet they provided informative amplicons for array fabrication.

\section{Discrimination of gene isoforms by ExprAlign}

We have explored the extent to which ExprAlign supplements sequence alignment in distinguishing isoforms with gene families. Figure 5 (and Additional File 1, Section B) shows for a number of co-expression mountains the changes in transcript expression of constituent clones as a conventional heatmap across all three experimental conditions.

\section{(i) Fructose-bisphosphate aldolases}

Vertebrates possess three tissue-specific isoforms of fructose-bisphosphate aldolase [26-28]: A (muscle and red blood cells), B (liver, kidney, stomach and intestine) and C (brain, heart and ovary) [29]. Assembly of our common carp ESTs identified these three 3 isoforms: S341 (the prefix ' $S$ ' signifies an assembled sequence group from carpBASE 2.1; aldolase A), S488 (aldolase B) and S698 (aldolase C).

Aldolase A and B were separately resolved into two neighboring mountains: GE17b containing only S341 (isoform A) and GE33 containing only $\mathrm{S} 488$ (isoform B). The close proximity of these mountains in Figure 2 was indicative of the similarity of their expression patterns (see Figure 5), yet ExprAlign cleanly resolved even these subtly different expression patterns in precise agreement with the sequence alignments. Figure 5 shows that resolution between isoforms depended mainly on expression differences in the chronic cold-exposure experiment, and in just 3 tissues; brain, gill, heart and kidney. Thus, aldolase A was up-regulated not only in hypoxia muscle $\left(17^{\circ} \mathrm{C}\right)$ but also in hypoxia liver, hypoxia brain, hypoxia intestine $\left(30^{\circ} \mathrm{C}\right)$, cold brain, cold gill, cold heart, cold intestine and cold kidney. Aldolase B was up-regulated in cold-intestine, cold heart, hypoxia muscle $17^{\circ} \mathrm{C}$, hypoxia intestine $30^{\circ} \mathrm{C}$ and hypoxia liver. Previous work [29] compared the absolute level of the gene expression of aldolase isoforms, but our study focused on stress-regulated changes.

\section{(ii) Parvalbumins}

carpBASE2.1 contained 89 clones identified as parvalbumin. All of these were located in 8 entirely separate clusters that were positioned around the edge of the landscape (Figure 2a: P1 to P8), indicating very divergent expression patterns. Two distinct phylogenetic lineages for parvalbumins have previously been delineated: $\alpha$ and $\beta[30,31]$, and manual sequence alignment a single $\alpha$ isoform $(\alpha 1)$ and $8 \beta$ isoforms, $(\beta 1-\beta 8)$, all of which are expressed as proteins [32]. The $\beta 6$ isoform was located in P7 (GE16, see Figure $2 \mathrm{~b})$, P6 included $\beta 7$, P3 included $\beta 5$, whilst P1 (GE14b) contained $\beta 6, \beta 7$ and $\beta 1$.

\section{(iii) Transferrin variant $A$}

Crucian carp (C. auratus) has two transferrin variants [33], whilst silver crucian carp (C. auratus gibelio) has 5 and white crucian carp (C. auratus cuvieri) has 3 [34]. We found that ESTs blasting to transferrin variant A were located in two mountains, GE13 and GE17 that were located in two separate locations but in the same sector of the landscape. Whilst overall the expression profiles (Figure 5) were similar differences were noted in intestinal and cardiac tissues exposed to hypoxia. However, sequence alignment of the ESTs in these two mountains failed to demonstrate any sequence clustering which related to the separate expression mountains. The lengths of the GenBank accessions for carp transferrin variants (AF457152, EU71532-EU71535) were over 2200 bp whilst our ESTs were 400 bp of which only 200 bp could be used for sequence alignment. So it is most likely that the mountains contain different isoforms and that the sequence domains linked to these expression differences lay outside of the sequenced stretches.

\section{(iv) Apolipoproteins}

The apolipoprotein gene family were represented in five different mountains, namely GE11 (containing apolipoprotein C-I precursor, apoC-I), GE14 (apolipoprotein Eb precursor, apo-Eb), GE35 (apolipoprotein A-I, apoA-1), GE125 (14-kDa apolipoprotein, apo-14 kDa) and GE79 (mixed apolipoprotein \& its precursor). Pufferfish apoA-I was expressed mainly in liver but apo-14 kDa was mainly 


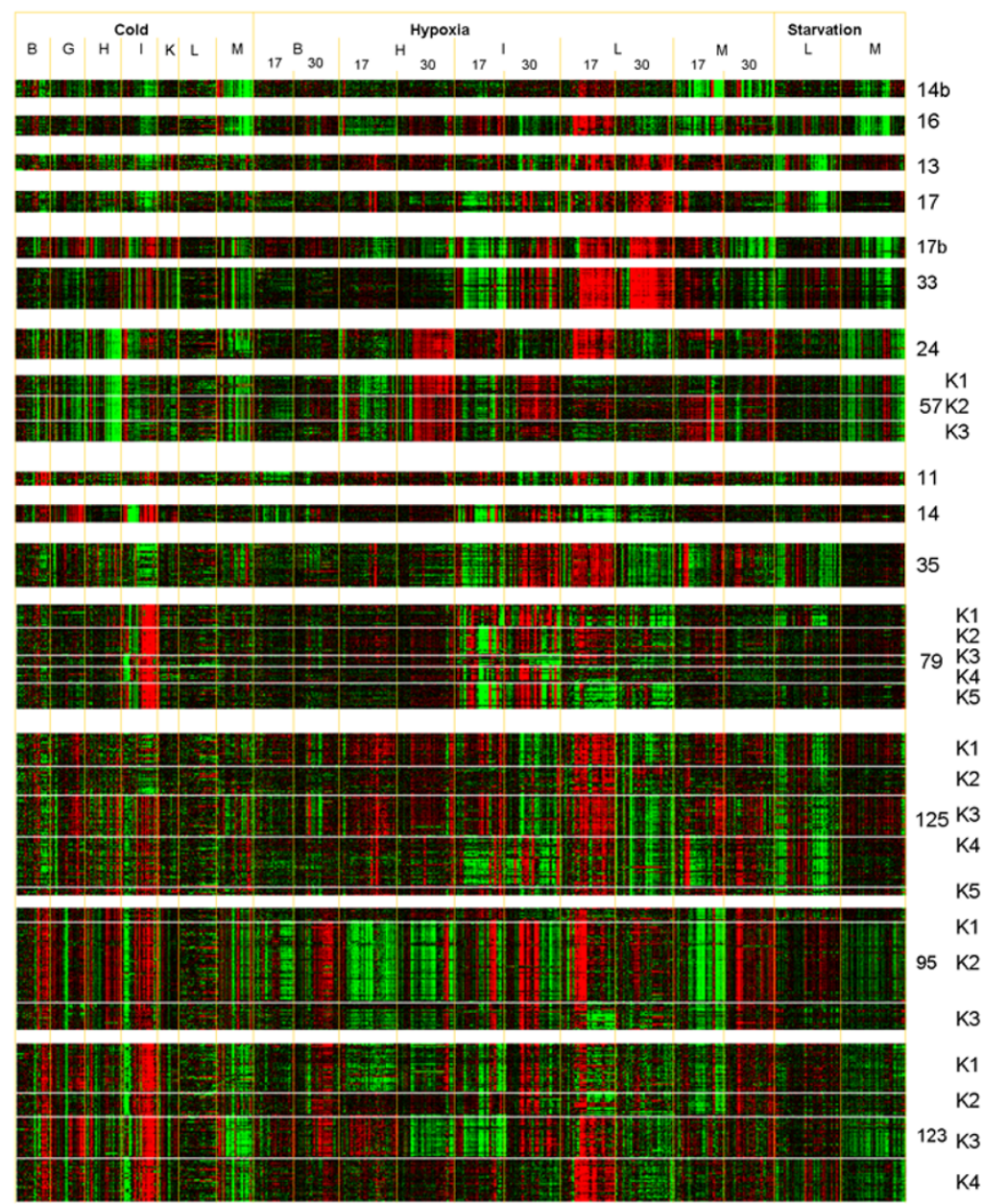

Figure 5

Heatmaps for identified GE mountains incorporating data for 707 RNA preparations comprising stress treatments in up to 7 different tissues. The numbers on the right side indicates the mountain names which corresponds to the number of gene probes. $\mathrm{KI}, \mathrm{K} 2, \cup, \mathrm{Kn}$ shows the sub-clusters generated by the $\mathrm{K}$-means clustering technique for each mountain. B - brain, G - gill, H - heart, I intestine, K kidney, L liver, and M - muscle. Red and green indicates up- and down-regulated gene expressions respectively. Heatmaps for additional identified GE mountains are shown in Additional File I, Figure S3.

expressed in liver and less abundantly in brain [35]. We show in common carp that some members of this gene family displayed hypoxia responses notably in the intestine (GE35, Figure 4). By contrast, the features GE11 and GE14 showed stress responses of apoC-I and apo-Eb genes in brain and intestine, respectively. The transcripts of the latter gene were also observed in gill.

$\sim 85 \%$ of BLAST-identified clones in GE79 were apolipoproteins (Table 1). Using the K-means method we subclustered the gene probes within GE79 into 5 clusters (K1 to K5, see Figure 5) which were linked to specific gene identities; thus, GE79-K3 was related to fatty acid-binding protein, GE79-K1 to apoA-I, GE79-K4 to apoEb precursor, and GE79-K2 and GE79-K5 were related to apoA-IV precursors. Mountain GE125 was split into $4 \mathrm{~K}$-means sub- clusters: GE125-K3 and GE125-K4 were 14 kDa (fishspecified) apolipoproteins, GE125-K2 was related to other apolipoproteins, and GE125-K1 was unknown.

\section{(v) Creatine kinases}

Creatine kinases were located in mountains GE57 and GE24. Vertebrates typically express four isoforms: cytosolic muscle type (M-CK), cytosolic brain type (B$\mathrm{CK}$ ), mitochondrial ubiquitous, acidic type (Miu-CK), and mitochondrial sarcomeric, basic type (Mis-CK) and three M-CK sub-isoforms (M1-CK, M2-CK, and M3-CK) have been reported for common carp [36], and were confirmed proteomically [37]. K-means clustering split GE57 into 3 sub-clusters; GE57-K2 was designated M2-CK, and GE57-K3 as M3-CK. GE57-K1 contained unidentified ESTs, which might be M1 or other isoform. 


\section{Discussion}

Approximately $46 \%$ of the singletons and assembled contigs in our common carp EST project failed to yield an identity using the unattended BLASTx procedure [10], some of which represent non-overlapping, or 3' sequences of identified genes. Other clones failed to yield a sequence on automated analysis yet provided suitable hybridisation probes on the microarray. Given these special problems and complications in this species due to a recently duplicated genome, the sometimes incoherent nature of subsequent gene losses [24], and the divergent tissue- and response-specific expression patterns so generated [9], we have explored how expression alignment techniques might complement the more usual sequence alignment methods to assign an identity for an otherwise unidentifiable sequence.

Our approach was based on the idea that expression profiles for non-overlapping probes derived from a same gene should be highly correlated when tested across a range of experimental treatments, and this should enable unidentified clones to be identified by comparison with identified clones. Similarly, comparing expression profiles for cDNA microarray probes possessing the same BLASTx identity, offers a means of testing their common identity, given that they may represent unrecognised isoforms or variants of a given gene. Thus, combining alignment of sequence data with that from gene expression data offers a useful means of improving the quality of gene identification, and for discriminating isoforms or members of gene families whose separate identify may not easily be made evident using conventional methods.

For this work we chose to include all available cDNA clones on the microarray, resulting in up to 80 clones per contig, and to gather expression profiles from a wide range of major organs and tissues, exposed to a range of experimental treatments. Consequently, the carp array included the substantial repetition of some genes and this provided greater support for the identified gene clusters. Our approach was based on the comparison of the expression profiles of pairs of probes using Pearsons correlation coefficients which were used to create a network linking genes together on the basis of their shared expression properties. The VxInsight algorithm uses a force-repulsion mechanism to gather the distributed gene networks into discrete clusters, which are then presented in an easy-tounderstand landscape metaphor.

We show that the resulting landscape features, and the associated clusters were robust, first, because permuting and randomising the expression values generated neither high correlation coefficients nor landscape features, and second, because the form of the clusters are largely retained when using different scales of array data from small to large. We show that datasets that contain a wider range of experimental treatments and tissues can fragment the gene clusters into smaller forms, each with a distinctive character. Thus, the exact level of discrimination achieved depends upon the diversity of the data used in its construction, with extra experimental treatments offering additional changes in the expression relationships between genes, thereby refining the resulting correlations.

\section{Gene identification using ExprAlign}

Many of the resulting landscape features or mountains generated by VxInsight contained predominantly just one kind of BLAST-identified gene, and we show that there is substantial enrichment of these genes within the features compared to chance alone. Thus unidentified probes within that mountain were also tentatively labelled with that gene identity. Using this approach we were able to impute an identity to 522 unidentified clones in the GE landscape, which represented $\sim 17 \%$ of all unidentified clones on the map. The validity of this assignment can be tested by the attended analysis of the clone sequences. This was achieved for mountain GE10 which possessed 5 different probes identified as myoglobin by BLASTx, and another 5 probes lacking an identify. Closer inspection of the corresponding sequences, and manual attempts at alignment, were subsequently able to demonstrate that all of the unidentified ESTs were also myoglobin, including additional examples of a unique brain-specific isoform [11]. This indicates the limitations of unattended BLAST analysis, and where appropriate, the need for manual verification of clones assigned with an ExprAlign identity. A similar result was obtained for parvalbumin [32].

\section{Separation of isoforms using ExprAlign}

ExprAlign has also proved useful in separating clones that have been assigned a common BLASTx identity but which have distinctive expression profiles. If clones possessing the same gene BLAST identity were indeed sourced from the same transcriptional start site, then they should display identical expression patterns. On the other hand, if the clones were representative of different isoforms with distinctive expression properties, then they would occupy different features on the expression landscape, or could be distinguished using additional clustering techniques. We show that these expectations are largely met for a series of test cases, as described above. As a further example, in the case of fatty acid-binding protein (mountain GE17c, not shown), we identified 13 cDNA clones all possessing the same BLASTx sequence alignment, but which displayed two contrasting expression profiles. In this particular case, the differences were very subtle and limited to just one tissue (liver) responding to just one treatment condition (hypoxia at $17^{\circ} \mathrm{C}$ ). Separation required application of $\mathrm{K}$ means clustering to the genes contained within the landscape feature. This again indicates that the level of func- 
tional dissection possible by ExprAlign depends to a significant extent on the diversity of treatments for which array data is generated.

\section{Conclusion}

By leveraging large-scale microarray data, the ExprAlign approach offers a practical aid to gene identification, and for the discovery of novel isoforms or variants of known genes. It bears comparison with the more conventional sequence alignment techniques in that both depend upon an association of co-expression properties, or sequence alignment, respectively, with a set of known standards. Both are limited by the available data, and both have limitations. We show that ExprAlign identifies subtle differences in expression properties which may not be evident from sequence similarity, particularly if the sequence data is limited to the 800 bp provided by a typical single pass read or if clones fail to generate sequence in automated procedures. Finally, it reveals ESTs possessing the same BLAST identity but which have distinctive expression profiles.

\section{Authors' contributions}

WL undertook the collation and analysis of microarray data and contributed to drafting the manuscript. LVM undertook sequence alignment and mapping onto zebrafish, ARC and AYG both conceived of the work, obtained funding, supervised the implementation and drafted the manuscript. $A B$ participated in the design of the study particularly to the form of the data analysis, the development of the ROC procedure, and the infrastructure for large-scale computations.

\section{Additional material}

\section{Additional file 1}

ExprAlign - the identification of ESTs in non-model species by alignment of cDNA microarray expression profiles. This document contains supplementary material for the abovementioned paper. It includes the following Sections - Section A - ROC curves to optimise thresholds for correlation score. Section B - Figures S1-S3. Section C - List of ESTs whose identity was inferred by ExprAlign procedure.

Click here for file

[http://www.biomedcentral.com/content/supplementary/14712164-10-560-S1.doc]

\section{Acknowledgements}

This work was supported by the Natural Environment Research Council (UK) to ARC and AYG.

\section{References}

I. Parkinson J, (ed): Expressed sequence tags: Generation and Analysis. Totowa, NJ.: Humana; 2009.

2. Mattick JS: A new paradigm for developmental biology. J Exp Biol 2007, 2 1 0: 1526-1547.
3. Carninci P: Constructing the landscape of the mammalian transcriptome. J Exp Biology 2007, 2 1 0: |497-I506.

4. Frith MC, Bailey TL, Kasukawa T, Mignone F, Kummerfeld SK, Madera M, Sunkara S, Furuno M, Bult C], Quackenbush J, Kai C, Kawai J, Carninci P, Hayashizaki Y, Pesole G, Mattick JS: Discrimination of nonprotein-coding transcripts from protein-coding mRNA. RNA Biol 2006, 3:40-48.

5. Cossins AR, Crawford DL: Fish as models for environmental genomics. Nat Rev Genet 2005, 6:324-333.

6. Larhammar D, Risinger C: Molecular genetic aspects of tetraploidy in the common carp Cyprinus carpio. Mol Phylogenet Evol 1994, 3:59-68.

7. David L, Blum S, Feldman MW, Lavi U, Hillel J: Recent duplication of the common carp (Cyprinus carpio L.) genome as revealed by analyses of microsatellite loci. Mol Biol Evol 2003, 20:1425-1434.

8. Robinson-Rechavi M, Marchand O, Escriva H, Bardet PL, Zelus D, Hughes S, Laudet V: Euteleost fish genomes are characterized by expansion of gene families. Genome Res 200I, I I:78I-788.

9. Gracey AY, Fraser EJ, Li W, Fang Y, Brass A, Rogers J, Cossins AR: Coping with cold: an integrative, multi-tissue analysis of the transcriptome of a poikilothermic vertebrate. Proc Natl Acad Sci USA 2004, I 0 I: I6970-16975.

10. Williams D, Li W, Hughes M, Gonzalez S, Vernon C, Vidall M, Jeney $Z$, Jeney G, Dixon P, McAndrew B, et al.: Genomic resources and microarrays for the common carp Cyprinus carpio L. J Fish Biol 2008, 72:2095-2II7.

II. Fraser J, Mello LV, Ward D, Rees HH, Williams DR, Fang Y, Brass A, Gracey AY, Cossins AR: Hypoxia-inducible myoglobin expression in nonmuscle tissues. Proc Natl Acad Sci USA 2006, I 03:2977-298I.

12. Stuart JM, Segal E, Koller D, Kim SK: A gene-coexpression network for global discovery of conserved genetic modules. Science 2003, 302:249-255.

13. Kim SK, Lund J, Kiraly M, Duke K, Jiang M, Stuart JM, Eizinger A, Wylie BN, Davidson GS: A gene expression map for Caenorhabditis elegans. Science 200I, 293:2087-2092.

14. Armstrong NJ, Wiel MA van de: Microarray data analysis: from hypotheses to conclusions using gene expression data. Cell Oncol 2004, 26:279-290.

15. McLachlan GJ, Do K, Ambroise C: Analyzing microarray gene expression data. A John Wiley \& Sons, Inc., Publication; 2004.

16. Kohonen T: Self-Organizing Maps. Berlin: Springer; 1995.

17. Jolliffe I: Principal Component Analysis. Springer; 2002.

18. Fang Y, Brass A, Hoyle DC, Hayes A, Bashein A, Oliver SG, Waddington $D$, Rattray $M$ : A model-based analysis of microarray experimental error and normalisation. Nucleic Acids Res 2003, 3 I :e96.

19. Anderson I, Brass A: Searching DNA databases for similarities to DNA sequences: when is a match significant. Bioinformatics 1998, 14:349-356.

20. Davidson GS, Hedrickson B, Johnson DK, Meyers CE, Wyle BN: Knowledge mining with VxInsight: discovery through interaction. J Intell Inf Sys 1998, I I :259-285.

21. Davidson GS, Wylie BN, Boyack KW: Cluster Stability and the Use of Noise in Interpretation of Clustering. Proc IEEE Symposium on Information Visualization 2001:23-30.

22. Fruchtermann T, Rheingold E: Graph drawing by force-directed placement, Technical Report UIUCDCS-R-90-1609. Computer Science, Univ. Illinois, Urbana-Champagne, II; 1990.

23. Christoffels A, Bartfai R, Srinivasan H, Komen H, Orban L: Comparative genomics in cyprinids: common carp ESTs help the annotation of the zebrafish genome. BMC Bioinformatics 2006, 7(S5):S2.

24. Evans H, De Tomaso T, Quail M, Rogers J, Gracey A, Cossins A, Berenbrink $M$ : Ancient and modern duplication events and the evolution of stearoyl-CoA desaturases in teleost fishes. Physiol Genomics 2008, 35: 18-29.

25. Cohen J: Statistical power analysis for the behavioral sciences. Second edition. Hillsdale, NJ: Lawrence Erlbaum Associates; 1988.

26. Perham RN: The fructose-I,6-bisphosphate aldolases: same reaction, different enzymes. Biochem Soc Trans 1990, 18:185-187

27. Marsh JJ, Lebherz HG: Fructose-bisphosphate aldolases: an evolutionary history. Trends Biochem Sci 1992, I 7: I I0- I I3. 
28. Shiokawa K, Kajita E, Hara H, Yatsuki H, Hori K: A developmental biological study of aldolase gene expression in Xenopus laevis. Cell Res 2002, I 2:85-96.

29. Shaw-Lee R, Lissemore JL, Sullivan DT, Tolan DR: Alternative splicing of fructose 1,6-bisphosphate aldolase transcripts in Drosophila melanogaster predicts three isozymes. J Biol Chem 1992, 267:3959-3967.

30. Elsayed $\mathrm{S}$, Bennich $\mathrm{H}$ : The primary structure of allergen $\mathbf{M}$ from cod. Scand I Immunol 1975, 4:203-208.

31. Lindstrom CD, Van do T, Hordvik I, Endresen C, Elsayed S: Cloning of two distinct cDNAs encoding parvalbumin, the major allergen of Atlantic salmon (Salmo salar). Scand J Immunol I 996, 44:335-344.

32. Brownridge P, Mello LV, Peters L, McLean L, A C, Cossins A, Whitfield $P$, Young $I$ : Regional variation in parvalbumin isoform expression correlates with muscle performance in Common Carp (Cyprinus carpio). J Exp Biology 2008, 21 2: 184- 193.

33. Yang L, Zhou L, Gui JF: Molecular basis of transferrin polymorphism in goldfish (Carassius auratus). Genetica 2004, | 2 1:303-313.

34. Yang L, Gui JF: Positive selection on multiple antique allelic lineages of transferrin in the polyploid Carassius auratus. Mol Biol Evol 2004, 21:1264-1277.

35. Kondo H, Morinaga K, Misaki R, Nakaya M, Watabe S: Characterization of the pufferfish Takifugu rubripes apolipoprotein multigene family. Gene 2005, 346:257-266.

36. Sun HW, Hui CF, Wu JL: Cloning, characterization, and expression in Escherichia coli of three creatine kinase muscle isoenzyme cDNAs from carp (Cyprinus carpio) striated muscle. J Biol Chem 1998, 273:33774-33780.

37. McLean L, Young I, Doherty M, Robertson D, Cossins A, Gracey A, Beynon R, Whitfield P: Global cooling: cold acclimation and the expression of soluble proteins in carp skeletal muscle. Proteomics 2007, 7:2667-2781.

Publish with Bio Med Central and every scientist can read your work free of charge

"BioMed Central will be the most significant development for disseminating the results of biomedical research in our lifetime. "

Sir Paul Nurse, Cancer Research UK

Your research papers will be:

- available free of charge to the entire biomedical community

- peer reviewed and published immediately upon acceptance

- cited in PubMed and archived on PubMed Central

- yours - you keep the copyright

Submit your manuscript here:

http://www.biomedcentral.com/info/publishing_adv.asp
BioMedcentral 